\title{
The Dark Matter and Dark Energy in Cosmic Evolution
}

\author{
Bin Liang ${ }^{1}$ \\ ${ }^{1}$ College of Science, Chongqing University of Posts and Telecommunication, Chongqing 400065, China \\ Correspondence: Bin Liang, College of Science, Chongqing University of Posts and Telecommunication, Chongqing \\ 400065, China. E-mail: liangbin@cqupt.edu.cn \\ Received: October 21, 2017 \\ Accepted: October 29, 2017 Online Published: November 1, 2017 \\ doi:10.5539/apr.v9n6p14 \\ URL: https://doi.org/10.5539/apr.v9n6p14
}

\begin{abstract}
This article is on an in-depth study of the relationship between the dark matter, dark energy, radiation field and gravitation field, based on the standard cosmic model and the principle that dark energy is originated from the destructive interference of electromagnetic radiation field. It explains the dynamic mechanism of cosmic expansion and contraction, and calculates the densities of dark matter, dark energy, radiation field and gravitation field.
\end{abstract}

Keywords: Gravitation field, radiation field, dark matter, dark energy, cosmic expansion and contraction

\section{Introduction}

In Liang (2015) we proved that the dark energy is originated from the destructive interference of electromagnetic radiation field, and that the increase of dark energy makes the universe expand. At the same time, the energy level of matter reduces, more and more matter becomes the dark matter with the dissipation of electromagnetic radiation field. The universe stops expanding and starts to contract in the action of gravity when the energy density of radiation field becomes small enough.

But the paper does not directly involve Einstein's equation of gravitational field. And this article will deeply study the relationship between dark matter, dark energy, radiation field and gravitation field, explain the dynamic mechanism of cosmic expansion and contraction, and calculate the densities of dark matter, dark energy, radiation field and gravitation field.

For ease of discussion, we need firstly to emphasize two basic concepts of the standard cosmic model: (1) the gravitational source is the massive matter and includes the dark matter; (2) the cosmic space is a finite space without boundary with the curvature $\mathrm{k}=1$.

We know that Einstein's equation of gravitational field (Weinberg, 1972) is

$$
R_{\mu \nu}=-8 \pi G\left(T_{\mu \nu}-\frac{1}{2} g_{\mu v} T\right)
$$

it degenerates into Newton's gravitational equation on the condition of static weak field, and Newton's gravitational equation is one about the massive matter, so, the pressure $p$ and density $\rho$ in the energy-momentum tensor

$$
T_{\mu \nu}=p g_{\mu \nu}+(\rho+p) U_{\mu} U_{v}
$$

should be the pressure and density of the massive matter, and they are functions of the cosmic time $t$.

The massive matter could be divided into two classes: visible matter and dark matter. Visible matter radiates the electromagnetic wave, and dark matter does not, like neutrinos and other massive particles in the quantum ground state. So

$$
\rho=\rho_{m}+\rho_{D m},
$$

where $\rho_{m}$ is the mass density of visible matter, $\rho_{D m}$ is that of dark matter.

Using Robertson-Walker metric (Weinberg, 1972) 


$$
d \tau^{2}=d t^{2}-R^{2}(t)\left[\frac{d r^{2}}{1-k r^{2}}+r^{2} d \theta^{2}+r^{2} \sin ^{2} \theta d \varphi^{2}\right]
$$

and Einstein's equation of gravitational field gives

$$
3 \ddot{R}=-4 \pi G(\rho+3 \mathrm{p}) R,
$$

and

$$
R \ddot{R}+2 \dot{R}^{2}+2 k=4 \pi G(\rho-\mathrm{p}) R^{2} .
$$

Eliminating $\ddot{R}$ from the two equations above gives the Friedmann equation:

$$
\dot{R}^{2}+\mathrm{k}=\frac{8 \pi G}{3} \rho R^{2} .
$$

Suppose there is $\dot{R}=0$ at some moment $t_{0}$, then

$$
\mathrm{k}=\frac{8 \pi G}{3} \rho\left(t_{0}\right) R^{2}\left(t_{0}\right)>0 .
$$

Since the curvature $\mathrm{k}$ is a constant, the equation above shows that it is impossible the curvature $\mathrm{k}$ equals 0 or -1 . And since there is $\mathrm{k}=1$ for $\rho R^{2}=3 / 8 \pi G$, so the cosmic space is a finite space without boundary with the curvature $\mathrm{k}=1$, and this means the universe experiences the lengthy expansion after the initial big bang, and then will experience a lengthy contraction and brew the next big bang, as is usually understood.

\section{Dark Matter Density}

The Friedmann equation (7) could be rewritten as

$$
\rho=\frac{3}{8 \pi G}\left(\mathrm{H}^{2}+\frac{\mathrm{k}}{R^{2}}\right),
$$

where the Hubble constant $H \equiv \dot{R} / R$. Substituting the equation above into equation (6) gives

$$
\begin{gathered}
p=-\frac{1}{3} \rho+\frac{2}{3} q_{0} \rho_{c}, \\
\rho_{c}=\frac{3 H^{2}}{8 \pi G}
\end{gathered}
$$

is usually called the critical mass density, and

$$
q_{0} \equiv-R \ddot{R} / \dot{R}^{2}
$$

is called the deceleration parameter. Substituting the present Hubble constant (Hinshaw et al., 2009) $H_{0}=74.3 \mathrm{~km} /(s \cdot M p c)$ into equation (11) gives

$$
\rho_{c}=\frac{3 H_{0}^{2}}{8 \pi G}=1.1 \times 10^{-26} \mathrm{~kg} / \mathrm{m}^{3} .
$$

Suppose the matter in the gravitational source is non-relativistic, the pressure $p \ll \rho$, take $q_{0} \approx 1$, the equation (10) gives

$$
\rho=2 \rho_{c} .
$$

According to the astronomical observation (Weinberg, 1972), the mass density in a galaxy, i.e. that of visible matter

$$
\rho_{m}=0.028 \rho_{c},
$$

so, the equation above and equation (3) give the mass density of dark matter:

$$
\rho_{D m}=1.972 \rho_{c} \gg \rho_{m} .
$$


The most direct evidence of existence of dark matter is the measurement of rotation speed of vortex galaxy (Li \& Xiao, 2000). Suppose the matter density is $\rho(\mathrm{r}) \approx B \frac{\rho_{D m}}{r^{2}}$ due to the gravitation in a vortex galaxy, where $r$ is the radius from the galaxy center, and $B$ is an undetermined constant, so the mass within the scope of $r$ is

$$
M(r)=\int_{0}^{r} \rho(r) 4 \pi r^{2} d r=4 \pi \rho_{D m} B r,
$$

and the rotation speed of the star at position $r$ is a constant independent of $r$ :

$$
v(r)=\sqrt{G \frac{M(\mathrm{r})}{r}}=\sqrt{4 \pi G B \rho_{D m}} .
$$

This is in line with astronomical observation and provides a way to determine the constant $B$. For example, if $v=150 \mathrm{~km} / \mathrm{s}$ (Weinberg, 1972) then there is

\section{Dark Energy Density}

$$
B=\frac{v^{2}}{4 \pi G \rho_{D m}}=1.24 \times 10^{45} / \mathrm{m} .
$$

In the standard cosmic model the energy conservation equation is (Weinberg, 1972)

$$
\dot{p} R^{3}=\frac{d}{d t}\left[R^{3}(\rho+p)\right],
$$

i.e.

$$
\dot{\rho}=-3(\rho+p) \frac{\dot{R}}{R}
$$

This shows that the source density decreases with cosmic expansion and increases with cosmic contraction. However, the equation above is obtained on the condition that galaxies neither are created nor disappear (Weinberg, 1972), and the actual situation is different.

In the cosmic lengthy evolution, the total energy should be conserved, although various kinds of energy wane and wax. At any time, if the universe doesn't expands, there should be

$$
\frac{d}{d t}\left(\rho+\rho_{e}+\rho_{G}+\rho_{d}\right)=0,
$$

where $\rho$ is the energy density of the gravitational source as mentioned before, $\rho_{G}$ is the energy density of the gravitational field, and $\rho_{d}$ is the dark energy density. Since the radiation field in the cosmic evolution is equivalent to the blackbody radiation field (Weinberg, 1972), the energy density of radiation field is $\rho_{e}=4 \sigma T^{4} / c$.

Without dissipation of the radiation field translating into dark energy due to destructive interference, radiation and absorption of electromagnetic waves is a conservative process, and $\frac{d}{d}\left(\rho+\rho_{e}\right)=0$. But because of the dissipation process of the radiation field translating into dark energy, there is

$$
\frac{d}{d}\left(\rho+\rho_{e}\right)=-\eta \rho_{e}
$$

where $\eta$ is the conversion rate from radiation field into dark energy per unit time (Liang, 2015).

Substituting the equation (23) into the equation (22) gives

$$
\eta \rho_{e}=\frac{d \rho_{d}}{d t}+\frac{d \rho_{G}}{d t} .
$$

This shows that a part of the dissipated energy of radiation field is translated into the dark energy and other is translated into the gravitational field.

Writing $f \equiv \frac{d \rho_{G}}{d t}$, the equation above can be rewritten as 


$$
\frac{d \rho_{d}}{d t}=\eta \rho_{e}-f,
$$

This is the equation (8) in the Liang (2015), but derived in a different way here.

Using $H \equiv \dot{R} / R$ the equation (11) in the paper can be rewritten as

$$
\frac{1}{R} \frac{\mathrm{d} R}{\mathrm{~d} t}=\frac{1}{\rho_{d}} \frac{\mathrm{d} \rho_{d}}{\mathrm{~d} t} .
$$

The two equations above show that when $\eta \rho_{e}>f$, dark energy increases and the universe expands; when $\eta \rho_{e}<f$, dark energy decreases and the universe contracts. This is the dynamical mechanism of cosmic expansion and contraction. From the equation above we know

$$
\rho_{d}=c_{d} R,
$$

where the proportional constant $c_{d}$ is determined by the latter equation (35).

\section{The Conversion Rate of Radiation Field and the Proportion of Dark Matter}

As shown in equation (3), the source of gravitational field includes visible and dark matter. On the cosmic time-space background, we can consider that the increase of dark matter equals the decrease of visible matter:

$$
\frac{d \rho_{D m}}{d t}=-\frac{d \rho_{m}}{d t} .
$$

Substituting the equation above and equation (3) into equation (23) gives

$$
\frac{d \rho_{e}}{d}=-\eta \rho_{e} .
$$

Suppose the conversion rate $\eta$ is a constant independent of time and integrating the equation above gives

$$
\eta=\frac{1}{\Delta t} \ln \left(\frac{\rho_{e}\left(t_{0}\right)}{\rho_{e}(t)}\right),
$$

where $\Delta t \equiv t-t_{0}>0$. Substituting $\rho_{e}=4 \sigma T^{4} / c$ into the equation above gives

$$
\eta=\frac{4}{\Delta t} \ln \left(\frac{T\left(t_{0}\right)}{T(t)}\right) .
$$

For instance, if $T\left(t_{0}\right)=4000 \mathrm{~K}$ (Weinberg, 1972), $T(t)=2.73 \mathrm{~K}, \Delta t=131.348 \times 10^{8} y$, then

$$
\eta=2.52 \times 10^{-9} / y=0.7984 \times 10^{-16} / s .
$$

The reason why the value above is different from that in Liang (2015) is that the calculation cited the equation $\rho_{d}=0.683 \rho_{c} c^{2}$ in the paper, but the equation is inaccurate.

As shown in equation (18), the rotation speed $v$ of a star at the position $r$ in a vortex galaxy is a constant independent of $r$, so the pressure of gravitation force is

$$
\rho_{D m} \frac{v^{2}}{r}
$$

and the work of dark energy to resist the gravitation force per unit time, i.e. the rate of change of gravitational energy is

$$
f=\frac{d \rho_{G}}{d t}=\rho_{D m} \frac{v^{2}}{R} \dot{R}=\rho_{D m} v^{2} H
$$

Substituting the equation above and equation (27) into equation (25) gives

$$
c_{d}=\frac{1}{\dot{R}}\left(\eta \rho_{e}-\rho_{D m} v^{2} H\right) .
$$

Thus the density of dark energy can be written as 


$$
\rho_{d}=\frac{\eta \rho_{e}}{H}-\rho_{D m} v^{2} .
$$

The present density of dark energy is

where

$$
\rho_{d}=\frac{\eta \rho_{e}}{H_{0}}-\rho_{D m} v^{2} \approx \frac{\eta \rho_{e}}{H_{0}}=1.42 \times 10^{-12} \mathrm{~J} / \mathrm{m}^{3}=1.43 \times 10^{-3} \rho_{c} c^{2} .
$$

If we define the energy density of gravitational field as

$$
\rho_{G} \equiv \rho_{D m} v^{2}=4.61 \times 10^{-16} \mathrm{~J} / \mathrm{m}^{3}=4.95 \times 10^{-7} \rho_{c} c^{2},
$$

from the three equations above we know that that $\rho_{d}, \rho_{e}$ and $\rho_{G}$ are negligible, and the total energy density of the universe can be written approximately as

$$
\rho_{0} \approx \rho_{m}+\rho_{D m} \approx 2 \rho_{c}
$$

So there is

$$
\Omega_{m}+\Omega_{D m} \approx 1
$$

where

$$
\begin{aligned}
& \Omega_{m}=\rho_{m} / \rho_{0} \approx 1.4 \%, \\
& \Omega_{D m}=\rho_{D m} / \rho_{0} \approx 98.6 \% .
\end{aligned}
$$

Obviously, the density of dark matter is far greater than that of visible matter in the present universe.

\section{Reference}

Bartelmann, M. (2010). The dark universe. Reviews of Modern Physics, 82(1), 331-382.

Bennett, C. L., Larson, D., Weiland, J. L., Jarosik, N., Hinshaw, G., Odegard, N., ... \& Komatsu, E. (2013). Nine-year Wilkinson Microwave Anisotropy Probe (WMAP) observations: final maps and results. The Astrophysical Journal Supplement Series, 208(2), 20. http://dx.doi.org/10.1088/0067-0049/208/2/20

Friedman, A. (1999). On the curvature of space. General Relativity and Gravitation, 31(12), 1991-2000. http://dx.doi.org/10.1007/BF01332580

Hinshaw, G., Weiland, J. L., Hill, R. S., Odegard, N., Larson, D., Bennett, C. L., ... \& Komatsu, E. (2009). Five-year wilkinson microwave anisotropy probe* Observations: data processing, sky maps, and basic results. The Astrophysical Journal Supplement Series, 180(2), 225.

Li, Z. W., \& Xiao, X. H. (2000). Astrophysics. Beijing: Higher Education Press.

Liang, B. (2015). The Origin of Dark Energy and Cosmic Expansion and Contraction. Applied Physics Research, 7(6), 134.

Peacock, J. A. (1999). Cosmological Physics. Cambridge: Cambridge University Press.

Peebles, P. J. E., \& Ratra, B. (2003). The cosmological constant and dark energy. Reviews of modern physics, 75(2), 559-606.

Perlmutter, S., Aldering, G., Goldhaber, G., Knop, R. A., Nugent, P., Castro, P. G., ... \& Hook, I. M. (1999). Measurements of $\Omega$ and $\Lambda$ from 42 high-redshift supernovae. The Astrophysical Journal, 517(2), 565-586. http://dx.doi.org/10.1086/307221

Riess, A. G., Filippenko, A. V., Challis, P., Clocchiatti, A., Diercks, A., Garnavich, P. M., ... \& Leibundgut, B. R. U. N. O. (1998). Observational evidence from supernovae for an accelerating universe and a cosmological constant. The Astronomical Journal, 116(3), 1009-1038. http://dx.doi.org/10.1086/300499

Weinberg, S. (2008). Cosmology. New York: Oxford University Press.

Weinberg, S. (1972). Gravitation and cosmology. John Wiley and Sons, New York.

\section{Copyrights}

Copyright for this article is retained by the author(s), with first publication rights granted to the journal.

This is an open-access article distributed under the terms and conditions of the Creative Commons Attribution license (http://creativecommons.org/licenses/by/4.0/). 